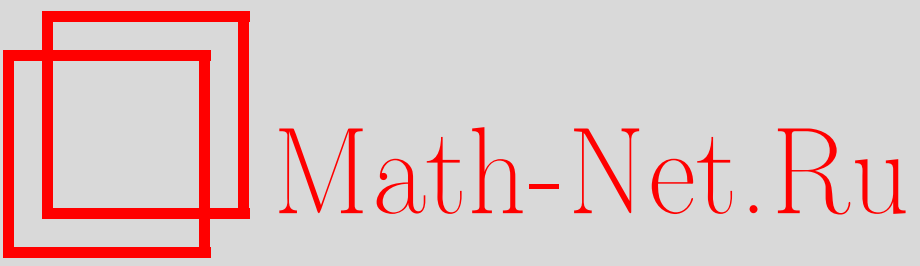

М. С. Маскина, М. И. Купцов, Частные случаи гиперболических параллелограммов на плоскости Лобачевского, Итоги науки и техн. Сер. Соврем. мат. и ее прил. Темат. обз., 2019, том 169, 39-47

DOI: https://doi.org/10.36535/0233-6723-2019-169-39-47

Использование Общероссийского математического портала Math-Net.Ru подразумевает, что вы прочитали и согласны с пользовательским соглашением

http: //www. mathnet.ru/rus/agreement

Параметры загрузки:

IP: 34.239 .49 .27

26 апреля 2023 г., 06:46:29 


\title{
ЧАСТНЫЕ СЛУЧАИ ГИПЕРБОЛИЧЕСКИХ ПАРАЛЛЕЛОГРАММОВ НА ПЛОСКОСТИ ЛОБАЧЕВСКОГО
}

\author{
(c) 2019 г. $\quad$ М. С. МАСКИНА, М. И. КУПЦОВ
}

\begin{abstract}
АннотАция. Рассмотрены частные случаи гиперболического параллелограмма, которые получены при перенесении на плоскость Лобачевского характеристических свойств прямоугольника и квадрата евклидовой плоскости, связанных с их диагоналями. Доказано существование этих четырехугольников на модели Кэли-Клейна в круге евклидовой плоскости.
\end{abstract}

Ключевые слова: плоскость Лобачевского, модель Кэли-Клейна, гиперболический параллелограмм, гиперболический ромб.

\section{SPECIAL CASES OF HYPERBOLIC PARALLELOGRAMS ON THE LOBACHEVSKY PLANE}

\author{
(c) 2019 M. S. MASKINA, M. I. KUPTSOV
}

\begin{abstract}
In this paper, we consider particular cases of hyperbolic parallelograms obtained by transferring to the Lobachevsky plane of characteristic properties of rectangles and squares on the Euclidean plane associated with their diagonals. The existence of these quadrangles in the CayleyKlein model in a circle of the Euclidean plane is proved.
\end{abstract}

Keywords and phrases: Lobachevsky plane, Cayley-Klein model, hyperbolic parallelogram, hyperbolic rhombus.

AMS Subject Classification: 51F99

1. Введение. Исторически сложилось, что основные типы четырехугольников плоскости Лобачевского принято классифицировать по наличию и количеству у них прямых углов: четырехугольники Ламберта, Нестеровича, Саккери (или первый четырехугольник Хайяма; см. [1-3]). Кроме четырехугольников, имеющих прямые углы, в литературе в качестве одних из наиболее важных типов четырехугольников плоскости Лобачевского упоминаются второй четырехугольник Хайяма (или равноугольный четырехугольник, см. [3]), правильный четырехугольник (или гиперболический квадрат, см. $[1,3])$, гиперболический параллелограмм и его частный случай гиперболический ромб (см. [1,3-5]). Остановимся подробнее на двух последних четырехугольникаx.

Гиперболическим параллелограммом называется четырехугольник, в котором противоположные стороны попарно параллельны (здесь и далее понимается параллельность по Лобачевскому). Вершина $B$, из которой выходят стороны $B A$ и $B C$, параллельные в данном направлении противоположным сторонам $C D$ и $A D$ соответственно, называется первой основной вершиной гиперболического параллелограмма, противоположная ей вершина $D$ - его второй основной вершиной, а две оставшиеся вершины $A$ и $C-$ боковыми вершинами (см. [3, с. 196]). 
Н. И. Лобачевский определил гиперболический параллелограмм, перенеся определение параллелограмма евклидовой плоскости на гиперболическую плоскость, поэтому мы поступили аналогичным образом с другими свойствами и признаками параллелограмма. Взяв некоторое характеристическое свойство параллелограмма евклидовой плоскости и перенеся его на гиперболическую плоскость в качестве определения, получим новый тип четырехугольника. Четырехугольник плоскости Лобачевского, обладающий некоторым характеристическим свойством параллелограмма евклидовой плоскости, назван нами квазипараллелограммом (см. [5]). В результате были сформулированы следующие десять определений четырехугольников плоскости Лобачевского, которые равносильны в плоскости Евклида:

$1^{\circ}$ квазипараллелограмм - это выпуклый четырехугольник, у которого две противоположные стороны параллельны и равны;

$2^{\circ}$ квазипараллелограмм - это выпуклый четырехугольник, противоположные стороны которого попарно равны;

$3^{\circ}$ квазипараллелограмм - это выпуклый четырехугольник, противоположные углы которого попарно равны;

$4^{\circ}$ квазипараллелограмм - это выпуклый четырехугольник, у которого диагонали точкой пересечения делятся пополам;

$5^{\circ}$ квазипараллелограмм - это выпуклый четырехугольник, у которого две противоположные стороны равны, а прямая, проходящая через их середины, образует с ними внутренние односторонние углы, сумма которых равна развернутому углу;

$6^{\circ}$ квазипараллелограмм - это выпуклый четырехугольник, у которого одна из диагоналей образует равные внутренние накрест лежащие углы с обеими парами противоположных сторон;

$7^{\circ}$ квазипараллелограмм - это выпуклый четырехугольник, у которого две противоположные стороны равны, а одна из диагоналей при пересечении с ними образует равные внутренние накрест лежащие углы;

$8^{\circ}$ квазипараллелограмм - это выпуклый четырехугольник, у которого каждая из диагоналей при пересечении с одной из пар противоположных сторон образует равные внутренние накрест лежащие углы;

$9^{\circ}$ квазипараллелограмм - это выпуклый четырехугольник, у которого две противоположные стороны равны, а третья сторона образует с ними внутренние односторонние углы, сумма которых равна развернутому углу;

$10^{\circ}$ квазипараллелограмм - это выпуклый четырехугольник, у которого каждая из двух пар углов, примыкающих к двум смежным сторонам, образует в сумме развернутый угол.

Было доказано, что определения $1^{\circ}, 2^{\circ}, 9^{\circ}$ и $10^{\circ}$ задают различные четырехугольники плоскости Лобачевского, которые мы назвали соответственно квазипараллелограммами I, II, III и IV родов, а определения $3^{\circ}-8^{\circ}$ равносильны определению $2^{\circ}$ (см. [5]). После рассмотрения различных определений параллелограммов естественно перейти к их частным случаям: ромбам, прямоугольникам и квадратам.

Гиперболическим ромбом Н. И. Лобачевский назвал гиперболический параллелограмм, у которого стороны, выходящие из одной из основных вершин, равны (см. [4]). Прямая, проходящая через основные вершины гиперболического ромба, является его осью симметрии (см. [1, с. 136]), а следовательно:

(1) его диагонали перпендикулярны,

(2) диагональ, проходящая через его основные вершины, является биссектрисой его внутренних углов,

(3) диагональ, проходящая через его боковые вершины, делится точкой пересечения диагоналей пополам,

(4) внутренние углы гиперболического ромба при боковых вершинах равны.

Более того, может быть доказано следующее утверждение.

Теорема 1. Если в гиперболическом параллелограмме диагонали перпендикулярны, то он является гиперболическим ромбом. 
Доказательство. Пусть $A B C D$ - гиперболический параллелограмм, диагонали которого перпендикулярны. Докажем, что $A B C D$ - гиперболический ромб. Рассмотрим семейство гиперболических параллелограммов с фиксированной стороной $A B=a$ и углом $A B C=\gamma$. Среди всех гиперболических параллелограммов данного семейства существует четырехугольник, диагонали которого перпендикулярны (это гиперболический ромб); докажем, что он единствен.

Предположим противное, т.е. что существует четырехугольник $A B C_{1} D_{1}$, отличный от $A B C D$, диагонали которого перпендикулярны. Тогда прямые $B A, C D$ и $C_{1} D_{1}$ принадлежат одному пучку параллельных прямых и возможны два случая:

(i) прямая $C D$ лежит между прямыми $B A$ и $C_{1} D_{1}$;

(ii) прямая $C_{1} D_{1}$ лежит между прямыми $B A$ и $C D$.

В первом случае образуется треугольник $A K O_{1}\left(K\right.$ - точка пересечения прямых $A C$ и $B D_{1}, O_{1}-$ точка пересечения прямых $A C_{1}$ и $\left.B D_{1}\right)$, для которого прямой угол $A O_{1} D_{1}$ является внешним; следовательно, $\angle A O_{1} D_{1}>\angle A K O_{1}$. Аналогично, $\angle A K O_{1}$ - внешний в треугольнике $B O K(O-$ точка пересечения прямых $A C$ и $B D)$; значит, $\angle A K O_{1}>\angle B O K$. Тогда $\angle A O_{1} D_{1}>\angle B O K$, что невозможно, так как оба угла прямые.

Во втором случае угол $B P O_{1}\left(P\right.$ - точка пересечения прямых $A C_{1}$ и $\left.B D\right)$ является внешним в треугольнике $A P O$, следовательно, $\angle B P O_{1}>\angle A O P$. Так как $\angle A O_{1} B$ внешний в треугольнике $B P O_{1}$, то $\angle A O_{1} B>\angle B P O_{1}$. Тогда прямой угол $A O_{1} B$ больше прямого угла $A O P$, что невозможно; значит, и этот случай не имеет места.

Таким образом, существует только один четырехугольник, принадлежащий вышеуказанному семейству, диагонали которого перпендикулярны, и им является гиперболический ромб $A B C D$. Теорема доказана.

Из теоремы 1 следует, что гиперболический ромб можно определить как гиперболический параллелограмм, диагонали которого перпендикулярны.

Продолжим рассмотрение частных случаев гиперболического параллелограмма, перенеся на плоскость Лобачевского характеристические свойства прямоугольника и квадрата евклидовой плоскости.

2. Гиперболический прямоугольник. В евклидовой плоскости прямоугольником называется параллелограмм, у которого все углы прямые. На плоскости Лобачевского такого четырехугольника не существует, так как сумма его углов будет равна $4 d$. Но из определения прямоугольника следует, что: (1) все его углы равны; (2) его диагонали равны. Первое утверждение, перенесенное на гиперболическую плоскость, дает равноугольный четырехугольник, или второй четырехугольник Хайяма, чьи противоположные стороны расходятся (см. [3]); следовательно, он не может быть частным случаем гиперболического параллелограмма. Поэтому введем в рассмотрение следующее определение гиперболического прямоугольника.

Определение 1. Гиперболическим прямоугольником будем называть гиперболический параллелограмм, диагонали которого равны.

Чтобы доказать, что гиперболический прямоугольник существует, рассмотрим модель плоскости Лобачевского в круге евклидовой плоскости (см. [2, с. 286]).

Пусть на евклидовой плоскости взята окружность $\omega$, заданная уравнением $x^{2}+y^{2}=1$. Возьмем во внутренней ее области три точки $A, B$ и $C$ общего положения, имеющие следующие координаты: $A(\bar{x} ; 0), B(0 ; 0), C(a ; b)$, где $a$ и $b$-произвольные числа, удовлетворяющие условию $a^{2}+b^{2}<1$. Наша задача состоит в том, чтобы при заданных $a$ и $b$ найти такое значение $\bar{x}$, что $|\bar{x}|<1$ и длины отрезков $A C$ и $B D$ равны по Лобачевскому.

Найдем координаты точки $D$ гиперболического прямоугольника $A B C D$. Так как на евклидовой плоскости уравнение прямой, заданной двумя точками с координатами $\left(x_{1} ; y_{1}\right)$ и $\left(x_{2} ; y_{2}\right)$, находится из условия

$$
\left|\begin{array}{cc}
x-x_{1} & x_{2}-x_{1} \\
y-y_{1} & y_{2}-y_{1}
\end{array}\right|=0
$$

то уравнения прямых $A B$ и $B C$ будут соответственно иметь вид $y=0$ и $y=b x / a$. 
Прямая $A B$ пересекает окружность $\omega$ в точках $M(1 ; 0)$ и $M^{\prime}(-1 ; 0)$. Координаты точек $N$ и $N^{\prime}$ пересечения прямой $B C$ с окружностью $\omega$ удовлетворяют системе уравнений

решив которую, получим

$$
y=\frac{b x}{a}, \quad x^{2}+y^{2}=1,
$$

$$
N\left(\frac{a}{\sqrt{a^{2}+b^{2}}} ; \frac{b}{\sqrt{a^{2}+b^{2}}}\right), \quad N^{\prime}\left(-\frac{a}{\sqrt{a^{2}+b^{2}}} ;-\frac{b}{\sqrt{a^{2}+b^{2}}}\right) .
$$

Введя обозначение $c=\sqrt{a^{2}+b^{2}}$, перепишем координаты точек $N$ и $N^{\prime}$ в виде $N(a / c ; b / c)$ и $N^{\prime}(-a / c ;-b / c)$.

Уравнения прямых $C M$ и $A N$ имеют соответственно вид

$$
y=b \frac{x-1}{a-1}, \quad y=b \frac{x-\bar{x}}{a-c \bar{x}} .
$$

Координаты точки $D$, как точки пересечения прямых $C M$ и $A N$, найдем, решив систему уравнений

$$
y=b \frac{x-1}{a-1}, \quad y=b \frac{x-\bar{x}}{a-c \bar{x}} .
$$

Получим, что точка $D$ имеет координаты

$$
D\left(\frac{a(\bar{x}-1)+\bar{x}(c-1)}{c \bar{x}-1} ; \frac{b(\bar{x}-1)}{c \bar{x}-1}\right) .
$$

Найдем условие равенства длин диагоналей $D B$ и $C A$. Длина отрезка $D B$ находится по формуле

$$
D B=\frac{r}{2}\left|\ln \left(D B, U_{1} U_{2}\right)\right|
$$

(см. [2, с. 329]), где $r>0$ - радиус кривизны пространства, $U_{1}$ и $U_{2}$ - точки пересечения прямой $D B$ с окружностью $\omega$. Найдем координаты точек $U_{1}$ и $U_{2}$ и сложное отношение $\left(D B, U_{1} U_{2}\right)$ :

$$
\left(D B, U_{1} U_{2}\right)=\frac{\left(D B, U_{1}\right)}{\left(D B, U_{2}\right)}=\frac{\lambda_{1}}{\lambda_{2}}
$$

где $\lambda_{1}\left(\lambda_{2}\right)$-отношение, в котором точка $U_{1}$ (соответственно, $\left.U_{2}\right)$ делит отрезок $D B$, т.е. $\lambda_{1}$ и $\lambda_{2}$ - числа, удовлетворяющие условиям $\overrightarrow{D U_{1}}=\lambda_{1} \overrightarrow{U_{1} B}$ и $\overrightarrow{D U_{2}}=\lambda_{2} \overrightarrow{U_{2} B}$. Из этих условий следует, что точки $U_{1}$ и $U_{2}$ имеют координаты

$$
U_{i}\left(\frac{a(\bar{x}-1)+\bar{x}(c-1)}{(c \bar{x}-1)\left(1+\lambda_{i}\right)} ; \frac{b(\bar{x}-1)}{(c \bar{x}-1)\left(1+\lambda_{i}\right)}\right), \quad i=1,2 .
$$

С другой стороны, $U_{1}$ и $U_{2}$ принадлежат окружности $\omega$. Значит, их координаты удовлетворяют уравнению $x^{2}+y^{2}=1$, т.е. $\lambda_{1}$ и $\lambda_{2}$ являются корнями уравнения

$$
\left(\frac{a(\bar{x}-1)+\bar{x}(c-1)}{(c \bar{x}-1)(1+\lambda)}\right)^{2}+\left(\frac{b(\bar{x}-1)}{(c \bar{x}-1)(1+\lambda)}\right)^{2}=1 .
$$

Это уравнение равносильно следующему уравнению:

$$
\lambda^{2}(c \bar{x}-1)^{2}+2 \lambda(c \bar{x}-1)^{2}+(c \bar{x}-1)^{2}-\Delta=0,
$$

где $\Delta=2 a \bar{x}(\bar{x}-1)(c-1)+c^{2}(\bar{x}-1)^{2}+\bar{x}^{2}(c-1)^{2}$. Отсюда

$$
\lambda_{1,2}=\frac{1-c \bar{x} \mp \sqrt{\Delta}}{c \bar{x}-1} .
$$

Таким образом,

Аналогично находим

$$
\left(D B, U_{1} U_{2}\right)=\frac{1-c \bar{x}-\sqrt{\Delta}}{1-c \bar{x}+\sqrt{\Delta}}
$$

$$
\left(C A, V_{1} V_{2}\right)=\frac{\left(C A, V_{1}\right)}{\left(C A, V_{2}\right)}=\frac{\mu_{1}}{\mu_{2}},
$$


где $\mu_{1}$ и $\mu_{2}$ - числа, удовлетворяющие условиям $\overrightarrow{C V_{1}}=\mu_{1} \overrightarrow{V_{1} A}$ и $\overrightarrow{C V_{2}}=\mu_{2} \overrightarrow{V_{2} A}$. Отсюда следует, что точки $V_{1}$ и $V_{2}$ имеют координаты

$$
V_{i}\left(\frac{a+\mu_{i} \bar{x}}{1+\mu_{i}} ; \frac{b}{1+\mu_{i}}\right), \quad i=1,2 .
$$

Так как $V_{1}$ и $V_{2}$ лежат на окружности $\omega$, то их координаты удовлетворяют уравнению $x^{2}+y^{2}=1$; следовательно, $\mu_{1}$ и $\mu_{2}$ являются корнями уравнения

$$
\left(\frac{a+\mu \bar{x}}{1+\mu}\right)^{2}+\left(\frac{b}{1+\mu}\right)^{2}=1
$$

После ряда тождественных преобразований получим равносильное уравнение

$$
\mu^{2}\left(1-\bar{x}^{2}\right)+2 \mu(1-a \bar{x})+1-c^{2}=0,
$$

корни которого равны

$$
\mu_{1,2}=\frac{a \bar{x}-1 \mp \sqrt{(1-a \bar{x})^{2}-\left(1-c^{2}\right)\left(1-\bar{x}^{2}\right)}}{\left(1-\bar{x}^{2}\right)} .
$$

Таким образом,

$$
\left(C A, V_{1} V_{2}\right)=\frac{a \bar{x}-1-\sqrt{(1-a \bar{x})^{2}-\left(1-c^{2}\right)\left(1-\bar{x}^{2}\right)}}{a \bar{x}-1+\sqrt{(1-a \bar{x})^{2}-\left(1-c^{2}\right)\left(1-\bar{x}^{2}\right)}} .
$$

Длины диагоналей $A C$ и $B D$ равны тогда и только тогда, когда выполняется одно из равенств $\left(D B, U_{1} U_{2}\right)=\left(C A, V_{1} V_{2}\right)$ или $\left(D B, U_{1} U_{2}\right)=1 /\left(C A, V_{1} V_{2}\right)$. Пусть, для определенности, $\left(D B, U_{1} U_{2}\right)=\left(C A, V_{1} V_{2}\right)$, т.е.

$$
\frac{1-c \bar{x}-\sqrt{\Delta}}{1-c \bar{x}+\sqrt{\Delta}}=\frac{a \bar{x}-1-\sqrt{(1-a \bar{x})^{2}-\left(1-c^{2}\right)\left(1-\bar{x}^{2}\right)}}{a \bar{x}-1+\sqrt{(1-a \bar{x})^{2}-\left(1-c^{2}\right)\left(1-\bar{x}^{2}\right)}} .
$$

После ряда тождественных преобразований получим следующее уравнение:

$$
\begin{aligned}
&(1-c \bar{x}) \sqrt{(1-a \bar{x})^{2}-\left(1-c^{2}\right)\left(1-\bar{x}^{2}\right)}= \\
&=(a \bar{x}-1) \sqrt{2 a \bar{x}(\bar{x}-1)(c-1)+c^{2}(\bar{x}-1)^{2}+\bar{x}^{2}(c-1)^{2}} .
\end{aligned}
$$

Взяв за основу равенство $\left(D B, U_{1} U_{2}\right)=1 /\left(C A, V_{1} V_{2}\right)$, получим уравнение

$$
\begin{aligned}
&(1-c \bar{x}) \sqrt{(1-a \bar{x})^{2}-\left(1-c^{2}\right)\left(1-\bar{x}^{2}\right)}= \\
&=-(a \bar{x}-1) \sqrt{2 a \bar{x}(\bar{x}-1)(c-1)+c^{2}(\bar{x}-1)^{2}+\bar{x}^{2}(c-1)^{2}} .
\end{aligned}
$$

Возведем обе части полученного уравнения в квадрат и преобразуем его:

$$
\begin{aligned}
(\bar{x}-1)\left[c^{2}(a \bar{x}-1)^{2}(\bar{x}-1)+2 a \bar{x}(c-1)(a \bar{x}-1)^{2}\right. & \left.+(\bar{x}+1)\left(c^{2}-1\right)(c \bar{x}-1)^{2}\right]+ \\
& +\bar{x}^{2}(c-1)^{2}(a \bar{x}-1)^{2}-(a \bar{x}-1)^{2}(c \bar{x}-1)^{2}=0 .
\end{aligned}
$$

Так как

то

$$
\bar{x}^{2}(c-1)^{2}(a \bar{x}-1)^{2}-(a \bar{x}-1)^{2}(c \bar{x}-1)^{2}=(a \bar{x}-1)^{2}\left[\bar{x}^{2}-1-2 c \bar{x}(\bar{x}-1)\right],
$$

$$
\begin{aligned}
(\bar{x}-1)(c-1)\left[2 a \bar{x}(a \bar{x}-1)^{2}+(\bar{x}+1)(c+1)(c \bar{x}-1)^{2}\right]+ & \\
& +(\bar{x}-1)(a \bar{x}-1)^{2}\left[c^{2}(\bar{x}-1)+\bar{x}+1-2 c \bar{x}\right]=0 .
\end{aligned}
$$

Так как

$$
(a \bar{x}-1)^{2}\left[c^{2}(\bar{x}-1)+\bar{x}+1-2 c \bar{x}\right]=(a \bar{x}-1)^{2}\left[\bar{x}\left(c^{2}-2 c+1\right)-\left(c^{2}-1\right)\right],
$$


TO

$$
\begin{aligned}
& (\bar{x}-1)(c-1) \bar{x}\left[2 a(a \bar{x}-1)^{2}+(c+1)(c \bar{x}-1)^{2}+(a \bar{x}-1)^{2}(c-1)\right]+ \\
& +(\bar{x}-1)(c-1)\left[(c+1)(c \bar{x}-1)^{2}-(a \bar{x}-1)^{2}(c+1)\right]=0 .
\end{aligned}
$$

Так как

$$
(c+1)(c \bar{x}-1)^{2}-(a \bar{x}-1)^{2}(c+1)=(c+1)\left(\bar{x}^{2}\left(c^{2}-a^{2}\right)+2 \bar{x}(a-c)\right),
$$

TO

$\bar{x}(\bar{x}-1)(c-1)\left[\bar{x}^{2}\left(c^{3}+c^{2}+2 a^{3}+a^{2} c-a^{2}\right)+\bar{x}\left(c^{3}-c^{2}-2 c-a^{2} c-5 a^{2}-2 a c+2 a\right)+2 a c-2 c^{2}+4 a\right]=0$, причем, так как $\bar{x} \neq 0$ и $\bar{x} \neq 1$, то это уравнение равносильно следующему:

$$
\bar{x}^{2}\left(c^{3}+c^{2}+2 a^{3}+a^{2} c-a^{2}\right)+\bar{x}\left(c^{3}-c^{2}-2 c-a^{2} c-5 a^{2}-2 a c+2 a\right)+2 a c-2 c^{2}+4 a=0 .
$$

Таким образом, мы доказали, что если вершины гиперболического прямоугольника $A B C D$ в данной интерпретации плоскости Лобачевского имеют координаты $A(\bar{x} ; 0), B(0 ; 0), C(a ; b)$, то четвертая вершина $D$ имеет координаты

$$
D\left(\frac{a(\bar{x}-1)+\bar{x}(c-1)}{c \bar{x}-1} ; \frac{b(\bar{x}-1)}{c \bar{x}-1}\right)
$$

где $\bar{x}$ является корнем уравнения (4).

Докажем теперь обратное утверждение: если на плоскости Лобачевского (на ее модели в круге евклидовой плоскости) вершины четырехугольника $A B C D$ имеют координаты $A(\bar{x} ; 0), B(0 ; 0)$, $C(a ; b)$, а координаты $D$ задаются формулой (5), где $c=\sqrt{a^{2}+b^{2}}$, а $\bar{x}$ - корень уравнения (4), то $A B C D$ является гиперболическим прямоугольником.

Действительно, из уравнения (4) следует выполнение одного из уравнений (2) или (3), каждое из которых влечет за собой равенство длин отрезков $A C$ и $B D$. Параллельность прямых $A B$ и $C D$ и прямых $B C$ и $A D$ следует из вида координат точки $D$. Следовательно, $A B C D-$ гиперболический прямоугольник.

Чтобы доказать существование гиперболического прямоугольника, достаточно для некоторых конкретных значений параметров $a$ и $b$ показать, что точка $A$ с координатами $(\bar{x} ; 0)$ лежит во внутренней области окружности $\omega$.

Пусть $a=1 / 3, b=\sqrt{14} / 6$; тогда $c=\sqrt{a^{2}+b^{2}}=1 / \sqrt{2}$. Подставив эти данные в уравнение (4), получим

что равносильно уравнению

$$
\bar{x}^{2} \frac{21 \sqrt{2}+50}{108}-\bar{x} \frac{41 \sqrt{2}+14}{36}+\frac{1+\sqrt{2}}{3}=0,
$$

$$
\bar{x}^{2}(21 \sqrt{2}+50)-3 \bar{x}(41 \sqrt{2}+14)+36(1+\sqrt{2})=0 .
$$

Решим его:

$$
\bar{x}_{1}=\frac{3(41 \sqrt{2}+14)-3 \sqrt{12 \sqrt{2}+2086}}{2(21 \sqrt{2}+50)}<1, \quad \bar{x}_{2}=\frac{3(41 \sqrt{2}+14)+3 \sqrt{12 \sqrt{2}+2086}}{2(21 \sqrt{2}+50)}>1,
$$

т.е. точка $A_{1}\left(\bar{x}_{1} ; 0\right)$ принадлежит внутренней области окружности $\omega$, а точка $A_{2}\left(\bar{x}_{2} ; 0\right)$ - нет.

Найдем теперь приближенное значение координат точки $D$ при $\bar{x}=\bar{x}_{1}$ и сумму их квадратов, чтобы убедиться в том, что и эта точка принадлежит внутренней области окружности $\omega$. Так как

$$
\left(\frac{a(\bar{x}-1)+\bar{x}(c-1)}{c \bar{x}-1}\right)^{2}+\left(\frac{b(\bar{x}-1)}{c \bar{x}-1}\right)^{2} \approx 0,467<1,
$$

то точка $D$ принадлежит внутренней области окружности $\omega$, а, значит, четырехугольник $A B C D$ является гиперболическим прямоугольником. 
3. Гиперболический квадрат. Квадрат на евклидовой плоскости определяется либо как прямоугольник, у которого все стороны равны, либо как ромб, у которого все углы равны. На плоскости Лобачевского равенство всех сторон четырехугольника определяет правильный четырехугольник, у которого, как и у второго четырехугольника Хайяма, противоположные стороны расходятся (см. [3]). Поэтому аналог квадрата евклидовой плоскости, являющийся частным случаем гиперболического параллелограмма, не может быть определен через равенство сторон или углов, но должен одновременно являться и гиперболическим ромбом и гиперболическим прямоугольником. Таким образом, получаем следующее определение.

Определение 2. Гиперболическим квадратом будем называть гиперболический параллелограмм, диагонали которого перпендикулярны и равны.

Из определения гиперболического квадрата следует, что он является частным случаем гиперболического прямоугольника, поэтому доказывать его существование вновь будем на интерпретации плоскости Лобачевского в круге евклидовой плоскости. Пусть в евклидовой плоскости окружность $\omega$ задана уравнением $x^{2}+y^{2}=1$. Построим внутри окружности гиперболический квадрат $A B C D$ таким образом, что точка пересечения его диагоналей совпадает с началом координат.

Тогда вершины гиперболического квадрата будут иметь координаты $A\left(a_{1} ; 0\right), B\left(0 ; b_{1}\right), C\left(a_{2} ; 0\right)$, $D\left(0 ; b_{2}\right)$, где $a_{i}, b_{i}(i=1,2)$ - числа, лежащие в промежутке $(-1 ; 1)$. Ясно, что числа $b_{1}$ и $b_{2}$ (так же, как и числа $\left.a_{1}, a_{2}\right)$ будут разного знака; более того, так как $A B C D$ является частным случаем гиперболического ромба, то вершины $A$ и $C$ симметричны относительно прямой $B D$, а, значит, $a_{2}=-a_{1}=-a$.

Уравнения прямых $A B, B C, C D$ и $A D$ имеют соответственно вид

$$
y=-\frac{b_{1} x}{a}+b_{1}, \quad y=\frac{b_{1} x}{a}+b_{1}, \quad y=\frac{b_{2} x}{a}+b_{2}, \quad y=-\frac{b_{2} x}{a}+b_{2} .
$$

Координаты точки $M$-точки пересечения прямых $A B$ и $C D$-удовлетворяют системе уравнений

решив которую, получим, что

$$
y=-\frac{b_{1} x}{a}+b_{1}, \quad y=\frac{b_{2} x}{a}+b_{2},
$$

$$
M\left(\frac{a\left(b_{1}-b_{2}\right)}{b_{1}+b_{2}} ; \frac{2 b_{1} b_{2}}{b_{1}+b_{2}}\right) .
$$

Аналогично, точка $N$ пересечения прямых $A D$ и $B C$ имеет координаты

$$
N\left(-\frac{a\left(b_{1}-b_{2}\right)}{b_{1}+b_{2}} ; \frac{2 b_{1} b_{2}}{b_{1}+b_{2}}\right) .
$$

С другой стороны, точки $M$ и $N$ лежат на окружности $\omega$, следовательно, их координаты удовлетворяют уравнению окружности $\omega$, т.е.

$$
\left(\frac{a\left(b_{1}-b_{2}\right)}{b_{1}+b_{2}}\right)^{2}+\left(\frac{2 b_{1} b_{2}}{b_{1}+b_{2}}\right)^{2}=1
$$

откуда следует, что $\left(b_{1}+b_{2}\right)^{2}-4 b_{1}^{2} b_{2}^{2}>0$ и

$$
|a|=\frac{\sqrt{\left(b_{1}+b_{2}\right)^{2}-4 b_{1}^{2} b_{2}^{2}}}{\left|b_{1}-b_{2}\right|} .
$$

Пусть прямая $B D$ пересекает окружность $\omega$ в точках $U_{1}$ и $U_{2}$; тогда длина диагонали $B D$ находится из условия (1) (см. [2, с. 329]). Найдем координаты точек $U_{1}$ и $U_{2}$ и сложное отношение $\left(B D, U_{1} U_{2}\right)$ :

$$
\left(B D, U_{1} U_{2}\right)=\frac{\left(B D, U_{1}\right)}{\left(B D, U_{2}\right)}=\frac{\lambda_{1}}{\lambda_{2}}
$$

где $\lambda_{1}\left(\lambda_{2}\right)$-отношение, в котором точка $U_{1}$ (соответственно, $\left.U_{2}\right)$ делит отрезок $B D$, т.е. $\lambda_{1}$ и $\lambda_{2}$ - числа, удовлетворяющие условиям $\overrightarrow{B U_{1}}=\lambda_{1} \overrightarrow{U_{1} D}$ и $\overrightarrow{B U_{2}}=\lambda_{2} \overrightarrow{U_{2} D}$. Следовательно, точки $U_{i}$ 
$(i=1,2)$ имеют координаты

$$
U_{i}\left(0 ; \frac{b_{1}+\lambda_{i} b_{2}}{1+\lambda_{i}}\right), \quad i=1,2 .
$$

С другой стороны, $U_{1}$ и $U_{2}$ принадлежат окружности $\omega$; значит, их координаты удовлетворяют уравнению $x^{2}+y^{2}=1$, т.е. $\lambda_{1}$ и $\lambda_{2}$ являются корнями уравнения

$$
\left(\frac{b_{1}+\lambda b_{2}}{1+\lambda}\right)^{2}=1 \quad \Rightarrow \quad \lambda_{1,2}=\frac{-\left(b_{1} b_{2}-1\right) \mp\left|b_{1}-b_{2}\right|}{b_{2}^{2}-1} .
$$

Таким образом,

Аналогично

$$
\left(B D, U_{1} U_{2}\right)=\frac{1-b_{1} b_{2}-\left|b_{1}-b_{2}\right|}{1-b_{1} b_{2}+\left|b_{1}-b_{2}\right|}
$$

$$
\left(A C, V_{1} V_{2}\right)=\frac{\left(A C, V_{1}\right)}{\left(A C, V_{2}\right)}=\frac{\mu_{1}}{\mu_{2}},
$$

где $\mu_{1}$ и $\mu_{2}$ - числа, удовлетворяющие условиям $\overrightarrow{A V_{1}}=\mu_{1} \overrightarrow{V_{1} C}$ и $\overrightarrow{A V_{2}}=\mu_{2} \overrightarrow{V_{2} C}$. Следовательно, так как точки $V_{1}$ и $V_{2}$ имеют соответственно координаты $\left(x_{1} ; 0\right)$ и $\left(x_{2} ; 0\right)$, получаем

$$
V_{i}\left(\frac{a-\mu_{i} a}{1+\mu_{i}} ; 0\right), \quad i=1,2 .
$$

Так как $V_{1}$ и $V_{2}$ лежат на окружности $\omega$, то их координаты удовлетворяют уравнению $x^{2}+y^{2}=1$, т.е. $\mu_{1}$ и $\mu_{2}$ являются корнями уравнения

$$
\left(\frac{a-\mu a}{1+\mu}\right)^{2}=1 \quad \Rightarrow \quad \mu_{1,2}=\frac{(|a| \mp 1)^{2}}{a^{2}-1}
$$

Таким образом,

$$
\left(A C, V_{1} V_{2}\right)=\frac{(|a|-1)^{2}}{(|a|+1)^{2}} .
$$

Длины диагоналей $A C$ и $B D$ равны тогда и только тогда, когда выполняется одно из равенств: $\left(B D, U_{1} U_{2}\right)=\left(A C, V_{1} V_{2}\right)$ или $\left(B D, U_{1} U_{2}\right)=\left(C A, V_{1} V_{2}\right)$. Пусть, для определенности, $\left(B D, U_{1} U_{2}\right)=$ $\left(A C, V_{1} V_{2}\right)$, т.е.

$$
\frac{1-b_{1} b_{2}-\left|b_{1}-b_{2}\right|}{1-b_{1} b_{2}+\left|b_{1}-b_{2}\right|}=\frac{(|a|-1)^{2}}{(|a|+1)^{2}} \text {. }
$$

Преобразовав это уравнение, получим:

$$
a^{2}\left|b_{1}-b_{2}\right|-2|a|\left(1-b_{1} b_{2}\right)+\left|b_{1}-b_{2}\right|=0 .
$$

Подставив в (7) выражение (6) и проведя несложные преобразования, получим

$$
b_{1}^{2}-2 b_{1}^{2} b_{2}^{2}+b_{2}^{2}+\left(b_{1} b_{2}-1\right) \sqrt{\left(b_{1}+b_{2}\right)^{2}-4 b_{1}^{2} b_{2}^{2}}=0 .
$$

Взяв за основу равенство $\left(B D, U_{1} U_{2}\right)=\left(C A, V_{1} V_{2}\right)$, мы получили бы вместо уравнения $(7)$ уравнение

$$
a^{2}\left|b_{1}-b_{2}\right|+2|a|\left(1-b_{1} b_{2}\right)+\left|b_{1}-b_{2}\right|=0,
$$

а после подстановки в него выражения (6) - уравнение

$$
b_{1}^{2}-2 b_{1}^{2} b_{2}^{2}+b_{2}^{2}-\left(b_{1} b_{2}-1\right) \sqrt{\left(b_{1}+b_{2}\right)^{2}-4 b_{1}^{2} b_{2}^{2}}=0 .
$$

Таким образом, мы доказали, что если $A B C D$ - гиперболический квадрат плоскости Лобачевского (на ее модели в круге евклидовой плоскости) с основными вершинами $B\left(0 ; b_{1}\right), D\left(0 ; b_{2}\right)$ и боковыми вершинами $A\left(a_{1} ; 0\right), C\left(a_{2} ; 0\right)$, то $b_{1}$ и $b_{2}$-различные числа, удовлетворяющие уравнению (8) или (9), $a_{2}=-a_{1}=-a$, а число $a$ находится по формуле (6). 
Докажем обратное утверждение: если различные числа $b_{1}$ и $b_{2}$, лежащие в промежутке $(-1 ; 1)$, удовлетворяют уравнению (8), а число $a$, найденное по формуле (6), меньше 1 , то четырехугольник $A B C D$ плоскости Лобачевского (при ее интерпретации в круге евклидовой плоскости), вершины которого имеют координаты $A(a ; 0), B\left(0 ; b_{1}\right), C(-a ; 0), D\left(0 ; b_{2}\right)$, является гиперболическим квадратом.

Действительно, если $b_{1}$ и $b_{2}$ удовлетворяют уравнению (8), то они удовлетворяют равносильному ему уравнению

$$
\frac{\left(b_{1}+b_{2}\right)^{2}-4 b_{1}^{2} b_{2}^{2}}{\left|b_{1}-b_{2}\right|}-2 \frac{\sqrt{\left(b_{1}+b_{2}\right)^{2}-4 b_{1}^{2} b_{2}^{2}}}{\left|b_{1}-b_{2}\right|}\left(1-b_{1} b_{2}\right)+\left|b_{1}-b_{2}\right|=0 .
$$

Введя новую переменную $а$, найденную по формуле (6), получим уравнение (7), которое, в свою очередь, равносильно уравнению

$$
\frac{1-b_{1} b_{2}-\left|b_{1}-b_{2}\right|}{1-b_{1} b_{2}+\left|b_{1}-b_{2}\right|}=\frac{(|a|-1)^{2}}{(|a|+1)^{2}} .
$$

Последнее уравнение означает равенство сложных отношений $\left(B D, U_{1} U_{2}\right)$ и $\left(A C, V_{1} V_{2}\right)$, что говорит о равенстве длин диагоналей в четырехугольнике $A B C D$. Перпендикулярность диагоналей следует из того, что противоположные вершины четырехугольника лежат на координатных осях, а параллельность противоположных сторон - из равенства (6).

Докажем существование гиперболического квадрата. Пусть $b_{1}=0,5$; тогда уравнение (8) примет вид

$$
0,25+0,5 b_{2}^{2}+\left(0,5 b_{2}-1\right) \sqrt{b_{2}+0,25}=0 .
$$

Рассмотрим функцию $f\left(b_{2}\right)=0,25+0,5 b_{2}^{2}+\left(0,5 b_{2}-1\right) \sqrt{b_{2}+0,25}$. На промежутке $[-0,25 ;+\infty)$ функция $f\left(b_{2}\right)$ непрерывна, причем $f(0)=-0,25$ и $f(-0,25)=9 / 32$. Следовательно, на интервале $(-0,25 ; 0)$ данная функция $f\left(b_{2}\right)$ по крайней мере в одной точке обращается в нуль. Приближенное значение этой точки равно $-0,190073$. Тогда из $(6)$ следует $|a| \approx 0,7898608<1$. Таким образом, все вершины четырехугольника $A B C D$ лежат во внутренней области окружности $\omega$ и, значит, $A B C D$ является гиперболическим квадратом.

Таким образом, сформулированы определения частных случаев гиперболического параллелограмма: гиперболических ромба, прямоугольника и квадрата, обладающих характеристическими свойствами соответствующих четырехугольников евклидовой плоскости, связанные с равенством или перпендикулярностью их диагоналей, и доказано существование этих четырехугольников.

\section{СПИСОК ЛИТЕРАТУРЫ}

1. Атанасян Л. С. Геометрия Лобачевского. - М.: Просвещение, 2001.

2. Атанасян Л. С., Базылев В. Т. Геометрия. - М.: Просвещение, 1987.

3. Каган В. Ф. Основания геометрии. - М.-Л.: ГИТТЛ, 1949.

4. Лобачевский Н. И. Полное собрание сочинений в 3 томах. - М.-Л.: ГИТТЛ, 1951.

5. Маскина M. C. Обучение доказательству математически одаренных учащихся на факультативных курcax/ Дисс. на соиск. уч. степ. канд. пед. наук. - Саранск: Мордов. гос. пед. ин-т им. М. Е. Евсевьева, 2003.

Маскина Мария Сергеевна

Академия права и управления Федеральной службы исполнения наказаний России, Рязань

E-mail: mariya_maskina@mail.ru

Купцов Михаил Иванович

Рязанский государственный радиотехнический университет

E-mail: kuptsov_michail@mail.ru 